\title{
Rotations associated with Lorentz boosts
}

\author{
S. Başkal ${ }^{1}$ \\ Department of Physics, Middle East Technical University, \\ 06531 Ankara, Turkey \\ Y. S. Kim ${ }^{2}$ \\ Department of Physics, University of Maryland, \\ College Park, Maryland 20742, U.S.A.
}

\begin{abstract}
It is possible to associate two angles with two successive non-collinear Lorentz boosts. If one boost is applied after the initial boost, the result is the final boost preceded by a rotation called the Wigner rotation. The other rotation is associated with Wigner's $O(3)$-like little group. These two angles are shown to be different. However, it is shown that the sum of these two rotation angles is equal to the angle between the initial and final boosts. This relation is studied for both low-speed and high-speed limits. Furthermore, it is noted that the two-by-two matrices which are under the responsibility of other branches of physics can be interpreted in terms of the transformations of the Lorentz group, or vice versa. Classical ray optics is mentioned as a case in point.
\end{abstract}

\footnotetext{
${ }^{1}$ electronic address:baskal@newton.physics.metu.edu.tr

${ }^{2}$ electronic address: yskim@physics.umd.edu
} 


\section{Introduction}

The Wigner rotation is known as a by-product of two successive Lorentz boosts in special relativity. The earliest manifestation of the Wigner rotation is the Thomas precession which we observe in atomic spectra. Thomas formulated this problem thirteen years before the appearance of Wigner's 1939 paper 1, 2. The Thomas effect in nuclear spectroscopy is mentioned in Jackson's book on electrodynamics 3. Indeed, the Wigner rotation is the key issue in many branches of physics involving Lorentz boosts [4].

Recently, the Lorentz group has become an important scientific language in both quantum and classical optics. The theory of squeezed states is a representation of the Lorentz group [5, 6. Optical instruments are unavoidable in modern physics, and they are based on classical ray optics. It is gratifying to observe that the Lorentz group, through its two-by-two representation, is the basic underlying scientific language for ray optics, including polarization optics [7, interferometers [8, lens optics [9, 10], laser cavities [1], and multi-layer optics [12].

It is possible to perform mathematical operations of the Lorentz group by arranging optical instruments. For instance, the group contraction is one of the most sophisticated operations in the Lorentz group, but it has been shown recently that this can be achieved through focal process in one-lens optics [10]. Since there are many mathematical operations in optical sciences corresponding to Lorentz boosts, the Wigner rotation becomes one of the important issues in classical and quantum optics.

If we perform two Lorentz boosts in different directions, the result is not a boost, but is a boost preceded or followed by a rotation. This rotation is commonly known as the Wigner rotation. However, if we trace the origin of this word, Wigner introduced the rotation subgroup of the Lorentz group whose transformations leave the four-momentum of a given particle invariant in its rest frame. The rotation can however change the direction of its spin. Indeed, Wigner introduced the concept of "little group" to deal with this type of problem. Wigner's little group is the maximum subgroup of the Lorentz group whose transformations leave the four-momentum of the particle invariant. If the particle is moving, we can go to the Lorentz frame where it is at rest, perform a rotation without changing the momentum, and then come back to the original Lorentz frame. These transformations leave the momentum invariant. We shall hereafter call this little-group rotation "WLG rotation."

The question then is whether the Wigner rotation, as understood in the literature, is the same as the WLG rotation. This question was raised by 
Han et al. in their paper on Thomas precession and gauge transformations, but they have not made any attempt to clarify this issue [13. The present authors raised this question again in their paper on laser cavities 11]. They first noted that the two-by-two matrix formulation of lens optics is a representation of the Lorentz group, and then showed that the light beam performs one little-group rotation as it goes through one cycle in the cavity. Then they showed that the Wigner rotation and the WLG rotation are different, but those rotation angles were related for the special case of the Thomas precession.

The purpose of this paper is to establish the same relation for the most general case. We establish the difference between those two angles, and then show that they satisfy a complementary relation. In spite of the simplicity in concept, the calculations of these angles are not trivial.

Every relativistic problem has two important limits. One is the nonrelativistic limit, and the other is the light-like limit where the momentum of the particles becomes infinitely large. We also study these angles and their relation for the two limiting cases.

We note that the $S L(2, C)$, the group of unimodular two-by-two matrices, is the universal covering group of the Lorentz group, having the same algebraic property as the four-by-four representation of the Lorentz group. Although, for completeness we have included the expressions of the four-byfour transformation matrices, needless to say, their two-by-two counterparts can be expressed in a much more compact way. Furthermore, and more important than that, within the $\operatorname{SL}(2, \mathrm{C})$ formalism these matrix calculations can be applied to the two-by-two beam transfer matrices and the two-bytwo lens matrices in classical ray optics. Indeed, our basic motivation for the present paper came from our experience in ray optics. Thus, the group $S L(2, C)$ provides not only a topological base for the Lorentz group, but also concrete calculational tools for various branches of physics.

In Sec. 2, we consider two different rotations associated with two successive non-collinear Lorentz boosts. One is the Wigner rotation, and the other is the rotation associated with Wigner's little group. It is shown that the addition of these two angles is equal to the angle between the direction of the first boost and the the final boost. In Sec. 3] using the two-by-two matrices, we explicitly calculate those angles in terms of the parameters of the initial Lorentz boosts. In Sec. 4. we give some illustrative examples to show the dependence of the angles on the boost parameters. In Sec. [5, we explain how special relativity and ray optics find a common mathematical ground through their two-by-two matrix formalism. 


\section{Two Different Angles}

In the literature, the Wigner rotation comes from two successive Lorentz boosts performed in different directions. If we boost along the $z$ axis first and then make another boost along the direction which makes an angle $\phi$ with the $z$ axis on the $z x$ plane as shown in Fig. 1 the result is another Lorentz boost preceded by a rotation. This rotation is known as the Wigner rotation in the literature.

In the metric $(t, z, x, y)$, the rotation matrix which performs a rotation around the $y$ axis by angle $\phi$ is

$$
R(\phi)=\left(\begin{array}{cccc}
1 & 0 & 0 & 0 \\
0 & \cos \phi & -\sin \phi & 0 \\
0 & \sin \phi & \cos \phi & 0 \\
0 & 0 & 0 & 1
\end{array}\right)
$$

and its inverse is $R(-\phi)$.

The boost matrix requires two parameters. One is the boost parameter, and the other is the angle specifying the direction. We shall use the notation

$$
B(\phi, \eta)
$$

as the matrix performing a boost along the direction which makes an angle of $\phi$ with the boost parameter $\eta$. The boost matrix along the $z$ direction takes the form

$$
B(0, \eta)=\left(\begin{array}{cccc}
\cosh \eta & \sinh \eta & 0 & 0 \\
\sinh \eta & \cosh \eta & 0 & 0 \\
0 & 0 & 1 & 0 \\
0 & 0 & 0 & 1
\end{array}\right) .
$$

If this boost is made along the $\phi$ direction, the matrix is

$$
B(\phi, \eta)=R(\phi) B(0, \eta) R(-\phi)
$$

and its inverse is $B(\phi,-\eta)$.

Let us start with a massive particle at rest whose four-momentum is

$$
P_{a}=(m, 0,0,0),
$$

where $m$ is the particle mass. If we apply the boost matrix $B(0, \eta)$ to the four-momentum it becomes

$$
P_{b}=m(\cosh \eta, \sinh \eta, 0,0) .
$$




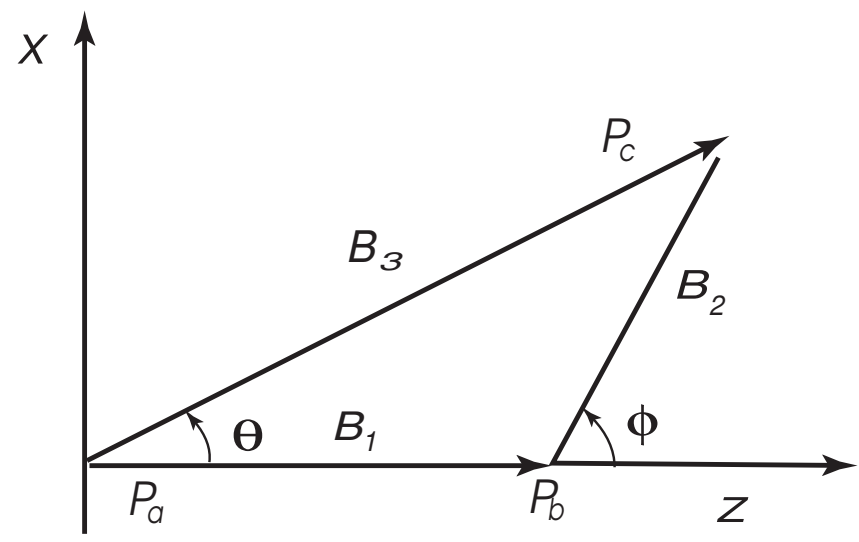

Figure 1: Two successive Lorentz boosts. Let us start from a particle at rest. If we make boost $B_{1}$ along the $z$ direction and another $B_{2}$ along the direction with makes an angle of $\phi$ with the $z$ direction, the net result is not $B_{3}$, but $B_{3}$ preceded by a rotation. This rotation is known as the Wigner rotation.

If we apply another boost $B(\phi, \lambda)$, the four-momentum takes the form

$$
P_{c}=m(\cosh \xi,(\sinh \xi) \cos \theta,(\sinh \xi) \sin \theta, 0) .
$$

The kinematics of these transformations is illustrated in Fig. 1] Then, we can consider the successive boosts

$$
B(\theta,-\xi) B(\phi, \lambda) B(0, \eta) .
$$

If this matrix is applied to $P_{a}$ of Eq.(15), it brings back to $P_{a}$. This means that the net effect is a rotation $R(\omega)$, which does not change the four-momentum of the particle in its rest-frame. This aspect is commonly written in the literature as

$$
B(\phi, \lambda) B(0, \eta)=B(\theta, \xi) R(\omega),
$$

where the matrices $B(0, \eta), B(\phi, \lambda)$ and $B(\theta, \xi)$ correspond to $B_{1}, B_{2}$ and $B_{3}$ in Fig. 11 respectively.

The product of the two boost matrices appears to be one boost matrix on the right-hand side in Fig. 1, but there must be a rotation matrix $R(\omega)$ to complete the mathematical identity. This rotation is known as the Wigner rotation in the literature.

$$
R(\omega)=B(\theta,-\xi) B(\phi, \lambda) B(0, \eta) .
$$




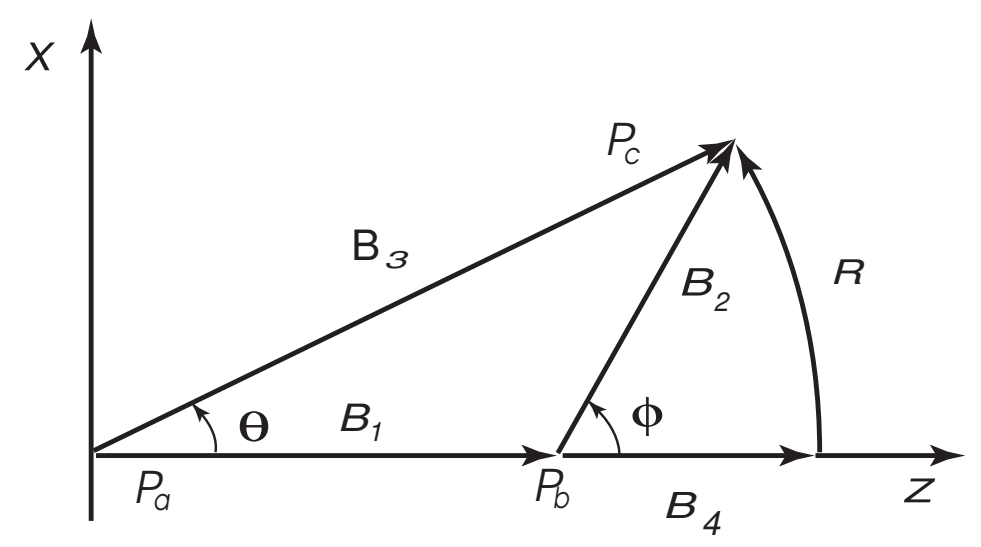

Figure 2: Closed Lorentz boosts. Initially, a massive particle is at rest with its four momentum $P_{a}$. The first boost $B_{1}$ brings $P_{a}$ to $P_{b}$. The second boost $B_{2}$ transforms $P_{b}$ to $P_{c}$. The third boost $B_{3}$ brings $P_{c}$ back to $P_{a}$. The particle is again at rest. The net effect is a rotation around the axis perpendicular to the plane containing these three transformations. We may assume for convenience that $P_{b}$ is along the $z$ axis, and $P_{c}$ in the $z x$ plane. The rotation is then made around the $y$ axis.

Let us consider a different transformation to obtain $P_{c}$ from $P_{b}$. We can first boost the system by $B(0, \xi-\eta)$, and rotate it by $R(\theta)$. The boost along the same direction does not change the helicity of the particle. The rotation $R(\theta)$ is also a helicity preserving transformation. This route is illustrated in Fig. 2. Helicity-conserving transformations has been discussed extensively in the literature [11, 14.

There are now two different ways of obtaining $P_{c}$ from $P_{b}$. If we choose the second route, and come back using $B(\phi,-\lambda)$, the net effect is

$$
D(\eta, \lambda, \phi)=B(\phi,-\lambda)[R(\theta) B(0, \xi-\eta)] .
$$

This transformation leaves the four-momentum $P_{b}$ given in Eq.(6) invariant. This "loop" transformation is illustrated in Fig.(3).

This is not the only way to leave the given the four-momentum unchanged. If we apply the boost $B(0,-\eta)$ to the four-momentum $P_{b}$ of Eq.(6), the result would be the four-momentum $P_{a}$ of Eq.(51). This is the four-momentum of the particle at rest. This four-momentum is invariant under three-dimensional rotations. This is precisely what Wigner observed in defining the $O(3)$-like rotation group for massive particles [2]. After performing a rotation which leaves $P_{a}$ invariant, we can boost the momentum 


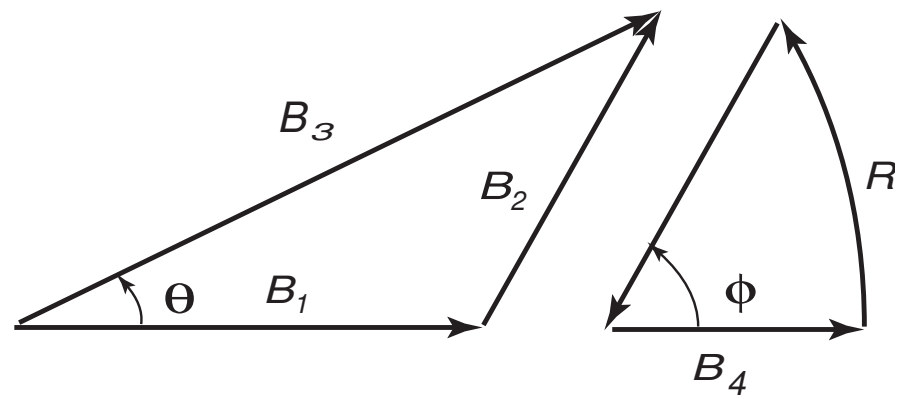

Figure 3: Addition of the angles. This figure consists of the Fig [ and the kinematics corresponding to the $D$ matrix of Eq.(11). This figure also illustrates the addition rule of Eq.(16).

back to $P_{b}$ by applying $B(0, \eta)$. The net effect is

$$
B(0, \eta) R(\alpha) B(0,-\eta) .
$$

This is the original definition of Wigner's little group which leaves $P_{b}$ invariant. The rotation matrix $R(\alpha)$ represents a three-dimensional rotation matrix.

We now demand that the little group transformation of Eq.(12) is the same as the $D$ matrix of Eq.(11). Then,

$$
B(0, \eta) R(\alpha) B(0,-\eta)=B(\phi,-\lambda) R(\theta) B(0, \xi-\eta) .
$$

This determines the angle $\alpha$ as:

$$
R(\alpha)=B(0,-\eta) B(\phi,-\lambda) R(\theta) B(0, \xi) .
$$

This is the WLG rotation angle as defined in Sec. 1.

Let us next consider the product $R(\omega) R(\alpha)$, where $R(\omega)$ and $R(\alpha)$ are from Eq.(10) and Eq.(14), respectively. Then

$$
R(\omega) R(\alpha)=R(\theta),
$$

which leads to

$$
\alpha+\omega=\theta .
$$

It is interesting to note that the above relation does not depend on the direction of the $B(\theta, \xi)$, nor does on the boost parameters $\eta$ and $\lambda$.

The purpose of this paper is to study consequences of the above relation. 


\section{Computation of the rotation angles}

In this section, we compute both Wigner rotation and WLG rotation angles. The two-by-two representation of the rotation matrix corresponding to the four-by-four expression of Eq.(11) is

$$
R(\phi)=\left(\begin{array}{cc}
\cos (\phi / 2) & -\sin (\phi / 2) \\
\sin (\phi / 2) & \cos (\phi / 2)
\end{array}\right)
$$

while the boost matrix given in Eq.(3) becomes

$$
B(0, \eta)=\left(\begin{array}{cc}
e^{\eta / 2} & 0 \\
0 & e^{-\eta / 2}
\end{array}\right)
$$

Let us use $B(\phi, \eta)$ for the boost along the direction which makes an angle $\phi$ with the $z$ axis. Then it takes the form

$$
\left(\begin{array}{cc}
\cosh (\eta / 2)+(\cos \phi) \sinh (\eta / 2) & (\sin \phi) \sinh (\eta / 2) \\
(\sin \phi) \sinh (\eta / 2) & \cosh (\eta / 2)-(\cos \phi) \sinh (\eta / 2)
\end{array}\right) .
$$

Uing these two-by-two expressions, we can complete all the computations for the transformation matrices given in Sec. 2 .

Let us go to the calculation of the Wigner rotation angle defined in Eq.(9). We can compute $\xi, \theta$ and $\omega$ in terms of $\eta, \lambda$ and $\phi$, by requiring that the right-hand side of Eq. (10) be a rotation matrix [15, 16]. The result of this calculation is

$$
\begin{aligned}
\cosh \xi & =\cosh \eta \cosh \lambda+\sinh \eta \sinh \lambda \cos \phi, \\
\tan \theta & =\frac{\sin \phi[\sinh \lambda+\tanh \eta(\cosh \lambda-1) \cos \phi]}{\sinh \lambda \cos \phi+\tanh \eta\left[1+(\cosh \lambda-1) \cos ^{2} \phi\right]}, \\
\tan \omega & =\frac{2(\sin \phi)\left[\sinh \lambda \sinh \eta+C_{-} \cos \phi\right]}{C_{+}+C_{-} \cos (2 \phi)+2 \sinh \lambda \sinh \eta \cos \phi},
\end{aligned}
$$

with

$$
C_{ \pm}=(\cosh \lambda \pm 1)(\cosh \eta \pm 1) .
$$

As for the angle $\alpha$, we first compute the boost parameter $\beta$ of $B_{4}$ in terms of $\eta, \lambda$ and $\phi$ as

$$
\tanh \beta=\frac{f-\tanh \eta(1+\tanh \eta \tanh \lambda \cos \phi)}{(1+\tanh \eta \tanh \lambda \cos \phi)-f \tanh \eta},
$$


and then obtain the $D$ matrix of Eq.(11) which takes the form

$$
D(\eta, \lambda, \phi)=\left(\begin{array}{cc}
{[(f+g) / 2 f]^{1 / 2}} & {\left[h_{+}(f-g) / 2 f\right]^{1 / 2}} \\
{\left[h_{-}(f-g) / 2 f\right]^{1 / 2}} & {[(f+g) / 2 f]^{1 / 2}}
\end{array}\right)
$$

where

$$
\begin{aligned}
& f=\frac{\sqrt{(\cosh \eta \cosh \lambda+\sinh \eta \sinh \lambda \cos \phi)^{2}-1}}{\cosh \eta \cosh \lambda}, \\
& g=\tanh \eta+\tanh \lambda \cos \phi, \\
& h_{ \pm}=\frac{1 \pm \tanh \eta}{1 \mp \tanh \eta} .
\end{aligned}
$$

The four-by-four counterpart of $D(\eta, \lambda, \phi)$ is of the form

$$
\left(\begin{array}{cccc}
{\left[f \cosh ^{2} \eta-g \sinh ^{2} \eta\right] / f} & n(g-f) / f & -\kappa / f & 0 \\
-n(g-f) / f & {\left[-f \sinh ^{2} \eta+g \cosh ^{2} \eta\right] / f} & -s / f & 0 \\
-\kappa / f & s / f & g / f & 0 \\
0 & 0 & 0 & 1
\end{array}\right)
$$

where

$$
\kappa=\tanh \eta \tanh \lambda \sin \phi, \quad n=\sinh \eta \cosh \eta, \quad s=\tanh \lambda \sin \phi .
$$

On the other hand, the left-hand side of Eq. (11) is $B(0, \eta) R(\alpha) B(0,-\eta)$, which takes the form

$$
\left(\begin{array}{cc}
\cos (\alpha / 2) & -e^{\eta / 2} \sin (\alpha / 2) \\
e^{-\eta / 2} \sin (\alpha / 2) & \cos (\alpha / 2)
\end{array}\right)
$$

Now, in view of Eq.(13), we can calculate the angle $\alpha$ by equating Eq.(23) and Eq.(27). The result is

$$
\tan \alpha=\frac{\tanh \lambda \sin \phi}{\sinh \eta+\cosh \eta \tanh \lambda \cos \phi} .
$$

We can check the addition law given in Eq.(16) by computing

$$
\tan (\alpha+\omega)=\frac{\tan \alpha+\tan \omega}{1-(\tan \alpha) \tan \omega} .
$$

After completion of this calculation using $\tan \omega$ and $\tan \alpha$ of Eq.(20) and Eq.(28) respectively, we end up with $\tan \theta$ of Eq.(20). 
In terms of the velocity of the particle, $\tanh \eta=v / c$. This means that $v=c \eta$ in the slow-speed limit. If the particle speed approaches the speed of light, $\tanh \eta$ becomes 1 . Let us consider the velocity additions in both cases. If $\eta$ and $\lambda$ are both small, the expressions in Eq.(20) become

$$
\begin{aligned}
& \xi^{2}=\eta^{2}+\lambda^{2}+\eta \lambda \cos \phi, \\
& \tan \theta=\frac{\lambda \sin \phi}{\eta+\lambda \cos \phi}, \\
& \alpha=\theta, \\
& \omega=0 .
\end{aligned}
$$

These expressions are consistent with the addition rules of non-relativistic kinematics. The Wigner rotation does not exist because $\omega=0$.

If $\eta$ and $\lambda$ are small, the system becomes the non-relativistic case. If $\eta$ becomes infinitely large, we are dealing with light-like particles. In the limit of large $\eta$ we have:

$$
\begin{aligned}
& \xi=\eta+\ln (\cosh \lambda+\sinh \lambda \cos \phi), \\
& \tan \theta=\frac{\sin \phi[\sinh \lambda+(\cosh \lambda-1) \cos \phi]}{\sinh \lambda \cos \phi+\left[1+(\cosh \lambda-1) \cos ^{2} \phi\right]}, \\
& \alpha=0, \\
& \omega=\theta .
\end{aligned}
$$

As for the $D$ matrix of Eq.(23), it becomes

$$
\left(\begin{array}{cc}
\cos (\alpha / 2) & -\sin (\alpha / 2) \\
\sin (\alpha / 2) & \cos (\alpha / 2)
\end{array}\right)
$$

in the limit of small $\eta$ and $\lambda$. This matrix represents a rotation by an angle $\alpha$ around the $y$ axis. This form is consistent with the expressions given in Eq.(27).

Let us go back to the original definition of Wigner's little group for massive particles. For a given massive particle, moving along the $z$ direction, we can bring the particle to its rest frame. Then we can perform a rotation without changing the four-momentum of the particle. However, the direction of its spin changes. We can bring back the particle to its original momentum by applying a boost matrix. This is what is happening in Eq.(12). If the 
amount of boost is very small, the little-group transformation is a rotation as given in Eq.(32).

For massless particles, it is not possible to bring the particle to its rest frame. The best we can do is to align the $z$ axis along the direction of the momentum. In his original paper [2, Wigner observed that the subgroup of the Lorentz group which dictates the internal space-time symmetry is locally isomorphic to the two-dimensional Euclidean group, with one rotational and two translational degrees of freedom. The rotational degree of freedom corresponds to the helicity, but the translation-like degrees were left unexplained.

Let us look at the $D$ matrix of Eq.(23). When $\eta$ becomes very large, and $\tanh \eta$ approaches 1 , this matrix becomes

$$
D(\lambda, \phi)=\left(\begin{array}{ll}
1 & u \\
0 & 1
\end{array}\right)
$$

where

$$
u=\frac{2 \tanh \lambda \sin \phi}{1+\tanh \lambda \cos \phi} .
$$

Similarly, when $\tanh \eta$ approaches to 1, the $D$ matrix of Eq.(25) becomes:

$$
D=\left(\begin{array}{cccc}
1+u^{2} / 4 & -u^{2} / 2 & -u & 0 \\
u^{2} / 2 & 1-u^{2} / 2 & -u & 0 \\
-u & u & 1 & 0 \\
0 & 0 & 0 & 1
\end{array}\right)
$$

This expression was given in Wigner's original paper [2], and corresponds to one of the translation-like transformations for the massless particle, but its physical interpretation as a gauge transformation was first given by Janner and Janssen [17. Indeed, this matrix had a stormy history [18, 19, 20], and its full story had not been told until 1990 when Kim and Wigner presented a cylindrical picture of the $E(2)$-like little group for massless particles 22]. This little group as a generator of gauge transformations is also an interesting subject in general relativity [21].

Furthermore, it is interesting to see that the expression of the $D$ matrix can be obtained as a large- $\eta$ limit of the Lorentz-boosted rotation of Eq.(27). This is a procedure known as the group contraction which Inönü and Wigner introduced to physics in 1953 [23. In their paper, Inönü and Wigner considered a two-dimensional plane tangent to a sphere, and observed that a small area on the spherical surface can be regarded as a two-dimensional 
plane with the two-dimensional Euclidean symmetry. Indeed, the InönüWigner contraction is the contraction of the rotation group $O(3)$ to the two-dimensional Euclidean group.

Since the symmetry groups for massive and massless particles are locally isomorphic to the rotation and Euclidean groups respectively, it was expected that the symmetry group of massless particle could be obtained through a contraction procedure. This aspect also has a history [24, 25, but the problem had not been completely clarified when Kim and Wigner in 1990 introduced a cylindrical symmetry for massless particles [22]. The question was that there are two-translational degrees of freedom while there is only one gauge degree of freedom.

\section{Illustrative Examples}

The calculations of Sec. 3 become simpler if the angle $\phi$ takes a special value. If this angle is such that the boost parameter $\xi$ remains the same as $\eta$, this transformation is responsible for Thomas precession. For this simpler case, the addition law $\theta=\alpha+\omega$ was noted in our earlier paper [11. The formulas of Eq.(20) and Eq.(28) become

$$
\begin{aligned}
\cosh \xi & =\cosh \eta \\
\tan \theta & =\tan \theta \\
\tan \alpha & =\frac{2 \sin \theta \cosh \eta}{\left.\sinh ^{2} \eta+\left(1+\cosh ^{2} \eta\right) \cos \theta\right)}, \\
\tan \omega & =\frac{\sin \theta\left[\cos \theta(\cosh \eta-1)^{2}+\sinh ^{2} \eta\right.}{\cos \theta\left[\cos \theta\left[(\cosh \eta-1)^{2}+\sinh ^{2} \eta\right]+2 \cosh \eta\right.} .
\end{aligned}
$$

In our earlier paper [11, we calculated $\alpha$ and $\omega$ in terms $\theta$ and $\eta$, instead of $\lambda$ and $\eta$.

If the angle $\phi$ is $90^{\circ}$, the expressions of Eq.(20) and Eq.(28) also become simpler, and the kinematics becomes quite transparent [26]. The angles are

$$
\begin{aligned}
& \cosh \xi=\cosh \eta \cosh \lambda, \\
& \tan \theta=\frac{\sinh \lambda}{\tanh \eta}
\end{aligned}
$$




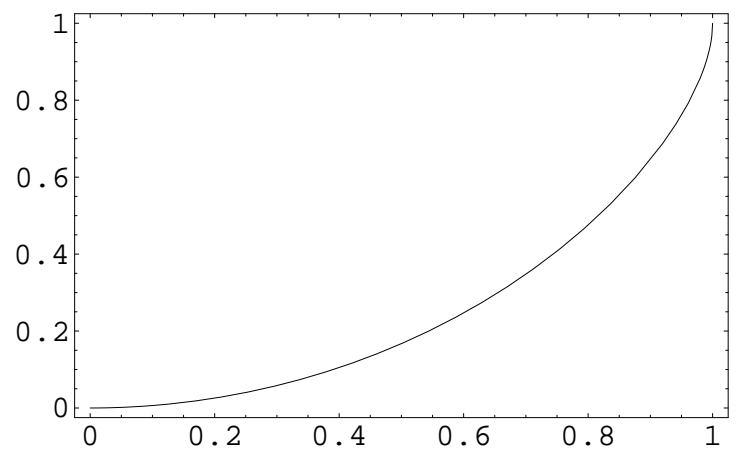

Figure 4: The ratio of the angle $\omega$ to the angle $\theta$ and a function of $\tanh \eta$, which becomes one as $\eta$ becomes very large. The ratio is zero at $\eta=0$, while it becomes one as $\eta$ approaches infinity. This was expected from the limiting cases discussed at the end of Sec 3 .

$$
\begin{aligned}
\tan \alpha & =\frac{\tanh \lambda}{\sinh \eta} \\
\tan \omega & =\frac{\sinh \lambda \sinh \eta}{\cosh \eta+\cosh \lambda} .
\end{aligned}
$$

We can now plot the above expressions as $\eta$ goes from zero to infinity, or as tanh $\eta$ goes from zero to 1 , for a given value of $\lambda$. Let us try the case with $\lambda=\eta$. Then the expressions become

$$
\begin{aligned}
& \cosh \xi=\cosh ^{2} \eta, \\
& \tan \theta=\cosh \eta, \\
& \tan \alpha=\frac{1}{\cosh \eta}, \\
& \tan \omega=\frac{\sinh \eta \tanh \eta}{2} .
\end{aligned}
$$

In terms of $\tanh \eta$,

$$
\begin{aligned}
& \cosh \xi=\frac{1}{1-\tanh ^{2} \eta} \\
& \tan \theta=\frac{1}{\sqrt{1-\tanh ^{2} \eta}}
\end{aligned}
$$




$$
\begin{aligned}
\tan \alpha & =\sqrt{1-\tanh ^{2} \eta} \\
\tan \omega & =\frac{\tanh ^{2} \eta}{2 \sqrt{1-\tanh ^{2} \eta}} .
\end{aligned}
$$

If we plot the angle $\theta$ against $\tanh \eta$, it starts with $45^{\circ}$ at $\eta=0$. The angle monotonically increases to $90^{\circ}$ as tanh $\eta$ reaches 1 . We can also plot $\alpha$ and $\omega$ to appreciate the addition rule given in Eq.(16).

\section{Physics of two-by-two matrices}

According to Eugene Wigner, quantum mechanics is the physics of Fourier transformations, and special relativity is the physics of Lorentz transformations.

In our recent papers, we formulated classical ray optics in terms of the two-by-two matrix representation of the Lorentz group, meaning that special relativity and ray optics has found a common mathematical formulation. It was noted that optical instruments can serve as analogue computers for special relativity through the use of those two-by-two matrices. Most of the calculations done in this present paper, particularly the group contraction mentioned in Sec. 3. can be carried out by optical instruments 10. Indeed, the motivation of this work is substantially based on the results of the papers written earlier by the present authors on ray optics.

Coherent and squeezed states in quantum optics can be formulated in terms of Wigner functions defined in two-dimensional phase space and linear canonical transformations [6]. Many physical theories are formulated as two-level problems. Most of the soluble models in physics take the form of coupled harmonic oscillators. Needless to say, all those diverse areas of physics are based on the mathematics of two-by-two matrices.

Einstein introduced his special relativity nearly one hundred years ago. This theory of course revolutionized our understanding of space and time, and thereby introduced to physics a mathematical device called the Lorentz group. Through its two-by-two representation, the Lorentz group is a very powerful instrument in theoretical physics.

\section{References}

[1] L. H. Thomas, Nature 117, 514 (1926); Philos. Mag. 3, 1 (1927). 
[2] E. P. Wigner, Ann. Math. 40, 149 (1939).

[3] J. D. Jackson, Classical Electrodynamics, Third Edition (Wiley, New York, 1999). See pages 552-53.

[4] H. Yamanaka, H. Matsumoto and H. Umezawa, Phys. Rev. D 24, 2607 (1981); F. Ciocci, G. Dattoli, C. Mari and A. Torre, Phys. Rev. A 46, 5149 (1992); B. Q. Ma, I. Schmidt and J. Soffer, Phys. Lett. B 441, 461 (1998); B. Q. Ma, I. Schmidt, Phys. Rev. D 58, 96008 (1998). A. Wambah, Nucl. Phys. B 434, 647 (1995); F. E. Close and A. Wambah, Nucl. Phys. B 412, 169 (1994); F. E. Close and A. Wambah, Phys. Lett. B 348, 207 (1995); F. E. Close and Z. P. Li, Phys. Lett. B 289, 143 (1992); J. A. Tjon and S. J. Wallace, Phys. Rev. C 44, 1156 (1991); G. Barenboim, J. Bernabeu and O. Vives, Phys. Rev. Lett. 77, 3299 (1996); K. Chen and C. Pei, Chem. Phys. Lett. 124, 365 (1986); B. J. Hare, K. P. Howard and J. H. Prestegard, Biophysical Journal 64, 392 (1993); H. Svensmark and H. C. Fogedby, Phys. Rev. E 49, R19 (1994); S. T. Lai, P. Palting, Y. N. Chiu and H. J. Silversone, J. Math. Chem. 24, 123 (1998); N. P. Benetis and A. R. Sornes, Concepts in Magnetic Resonance 12, 410 (2000).

[5] H. P. Yuen, Phys. Rev. A 13, 2226 (1976).

[6] Y. S. Kim and M. E. Noz, Phase Space Picture of Quantum Mechanics (World Scientific, Singapore, 1991).

[7] D. Han, Y. S. Kim, and M. E. Noz, Phys. Rev. E 56, 6065 (1997); D. Han, Y. S. Kim, M. E. Noz, Phys. Rev. E 60, 1036 (1999).

[8] D. Han, Y. S. Kim, and M. E. Noz, Phys. Rev. E 61, 5907 (2000).

[9] E. C. G. Sudarshan, N. Mukunda, and R. Simon, Optica Acta 32, 855 (1985); S. Başkal and Y. S. Kim, Phys. Rev. E 63, 056606 (2001).

[10] S. Başkal and Y. S. Kim, Phys. Rev. E 67, 056601 (2003).

[11] S. Başkal and Y. S. Kim, Phys. Rev. E 66, 026604 (2002).

[12] J. J. Monzón and L. L. Sánchez-Soto, Am. J. Phys. 64, 156 (1996); J. J. Monzón and L. L. Sánchez-Soto, J. Opt. Soc. Am. A, 17, 1475 (2000); J. J. Monzon and L. L. Sanchez-Soto, Journal of Modern Optics 48, 21 (2001); E. Georgieva and Y. S. Kim, Phys. Rev. E 64, 026602 (2001); J. J. Monzón, T. Yonte, L. L. Sánchez-Soto, and J. Carinena, J. Opt. 
Soc. Am. A, 19, 985 (2002); E. Georgieva and Y. S. Kim, Phys. Rev. E 68, 026606 (2003).

[13] D. Han, Y. S. Kim, and D. Son, Class. Quantum Grav. 4, 1777 (1987).

[14] D. Han, Y. S. Kim, and D. Son, Phys. Rev. D 31, 328 (1985).

[15] D. Han, Y. S. Kim, and M. E. Noz, Phys. Rev. A 37, 807 (1988).

[16] D. Han, E. Hardekopf, and Y. S. Kim, Phys. Rev. A 39, 1269 (1989).

[17] A. Janner and T. Janssen, Physica 53, 1 (1971); ibid. 60, 292 (1972).

[18] S. Weinberg, Phys. Rev. 134, B882 (1964); ibid. 135, B1049 (1964).

[19] D. Han and Y. S. Kim, Am. J. Phys. 49, 348 (1981). D. Han, Y. S. Kim, and D. Son, Phys. Rev. D 26, 3717 (1982).

[20] Y. S. Kim and M. E. Noz, Theory and Applications of the Poincaré Group (Reidel, Dordrecht, 1986).

[21] R. Banerjee and B. Chakraborty, Phys. Lett. B 502, 291 (2001); R. Banerjee and B. Chakraborty, J. Phys. A. Math.Gen. 35, 2183 (2002); T. Scaria and B. Chakraborty, Class. Quantum Grav. 19, 4445 (2002).

[22] Y. S. Kim and E. P. Wigner, J. Math. Phys. 31, 55 (1990).

[23] E. Inönü and E. P. Wigner, Proc. Natl. Acad. Sci. (U.S.) 39, 510 (1953).

[24] H. Bacry and N. P. Chang, Ann. Phys. 47, 407 (1968): S. Ferrara and C. Savoy, in Supergravity 1981, S. Ferrara and J. G. Taylor eds. (Cambridge Univ. Press, Cambridge, 1982), p. 151. P. Kwon and M. Villasante, J. Math. Phys. 29, 560 (1988); ibid. 30, 201 (1989).

[25] D. Han, Y. S. Kim, and D. Son, Phys. Lett. B 131, 327 (1983); D. Han, Y. S. Kim, M. E. Noz, and D. Son, Am. J. Phys. 52, 1037 (1984); D. Han, Y. S. Kim, and D. Son, J. Math. Phys. 27, 2228 (1986).

[26] D. Han, Y. S. Kim, and D. Son, J. Math. Phys. 28, 2373 (1987). 